\title{
Adenosine and ATP: traffic regulators in the kidney
}

\author{
Wilhelm Kriz \\ Department of Anatomy and Cell Biology, University of Heidelberg, Heidelberg, Germany.
}

\begin{abstract}
Glomerular filtration in the kidney is a continuous process that acts in concert with tubular reabsorption to prevent derangements of body fluid composition. Filtration is regulated by systemic factors, but it is also controlled by an intrinsic mechanism based on the anatomical connection between the distal nephron and the glomerular arterioles. Facing the threat of urinary salt loss, this mechanism causes vasoconstriction and reduces filtration by generating adenosine through the hydrolysis of nucleotide precursors such as $5^{\prime}$-AMP and possibly ATP (see the related article beginning on page 634).
\end{abstract}

Each human kidney is composed of one million nephrons, each of which is an individual unit that functions in large part independently of the others (Figure 1). The glomerulus, where filtration of the blood occurs, is a vascular and epithelial structure whose function is highly regulated. Although a variety of local and systemic hormones contribute significantly to this regulation, the anatomy of the nephron suggests an additional mode of control. The distal tubule, when it emerges from the medulla, is adherent to the glomerulus at the point of the macula densa. Ever since the anatomy of the whole mammalian nephron was discovered, this connection has suggested that the glomerulus likely receives signals from the tubules that transport the filtered fluid, not just from systemic hormones.

The conglomerate of cells at the site of contact is called the juxtaglomerular apparatus (JGA), and it is a cytoarchitectural feature of all mammalian kidneys (Figure 2). The JGA consists of, first, a plaque of tubular cells, the so-called macula densa (MD), that is located within the end portion of the thick ascending limb (TAL) of the loop of Henle, mediating contact between the TAL and the vascular pole of its parent glomerulus. It consists of, second, the extraglomerular mesangium, which is composed of cells and matrix and fills the angle between the afferent and efferent glomerular arterioles and, third, the VSMCs at the vascu-

Nonstandard abbreviations used: A1AR, A1 adenosine receptor; JGA, juxtaglomerular apparatus; $\mathrm{MD}$, macula densa; TAL, thick ascending limb.

Conflict of interest: The author has declared that no conflict of interest exists.

Citation for this article: J. Clin. Invest. 114:611-613 (2004). doi:10.1172/JCI200422669. lar pole, including the renin-producing juxtaglomerular granular cells. This intimate and systematic juxtaposition of tubular epithelial cells and vascular cells within the same nephron has given rise to early speculations about a functional connection in which a signal related to the composition of the tubular fluid at the MD affects glomerular vascular tone and glomerular filtration rate (1). It has now become clear that the JGA serves two different functions: it regulates the flow resistance of afferent arterioles in the so-called tubuloglomerular feedback mechanism, and it participates in the control of renin synthesis and release from the granular cells in the afferent arteriole (2). Researchers originally assumed that the two responses might be related to each other in that renin released from the granular cells not only has systemic relevance, but locally triggers the formation of Ang II and thus is responsible for the afferent vasoconstriction as well; however, it now appears that the final activation of smooth muscle and granular effector cells occurs through largely independent pathways. Renin release from granular cells is the major source of systemic Ang II and thus plays an essential role in controlling extracellular volume and blood pressure, whereas the vasoconstriction of the afferent arteriole locally serves to modulate the filmechanisms, it is well established that a change in $\mathrm{NaCl}$ concentration in the tubular fluid at the MD causes a graded release of mediators that reach their target by diffusion, thus acting in a paracrine fashion. It is worth remembering that the extraglomerular mesangium that mediates contact between the MD and the effector cells is tration of the concerned nephron. For both not vascularized, so that the buildup of any paracrine agent would not be perturbed by blood flow.

With respect to renin release, the most likely paracrine mediators of this process are prostaglandin E2 and nitric oxide (3-5), whereas the strongest experimental evidence for the vasoconstrictor response favors the notion that purinergic mediators, either ATP or adenosine, are responsible for signal transduction. A study by Castrop et al. reported in this issue of the JCI addresses the question of a possible interaction between ATP and adenosine in eliciting MD-dependent vasoconstriction (6).

\section{Regulation of vascular tone by adenosine or ATP}

As has been discussed recently, the issue of whether ATP or adenosine act as paracrine mediators of the tubuloglomerular feedback response is controversial, and experimental evidence for either possibility needs to be reconciled $(7,8)$. The role of adenosine as a mediator of the tubuloglomerular feedback response was proposed originally by Osswald et al. (9), supported by the finding that nonspecific inhibitors of adenosine receptors as well as specific A1 adenosine receptor (A1AR) antagonists markedly attenuate tubuloglomerular feedback responses (10, 11). A1ARs are expressed in afferent arterioles and they cause vasoconstriction by $\mathrm{G}_{\mathrm{i}}$-dependent activation of phospholipase $\mathrm{C}$ and subsequent $\mathrm{Ca}^{2+}$ entry via voltageactivated $\mathrm{Ca}^{2+}$ channels (12). Most importantly, mice with targeted disruption of the A1AR gene lack tubuloglomerular feedback responses, which suggests that adenosine, as the natural ligand of these receptors, is a nonredundant component of the signaling pathway $(13,14)$.

On the other hand, it has been suggested that ATP may be a mediator of the tubuloglomerular feedback response, a notion that is based on the finding that nonspecific antagonists of $\mathrm{P} 2$ receptors interfere with autoregulation of afferent arteriolar resistance, a phenomenon that is in part mediated through the tubuloglomerular 


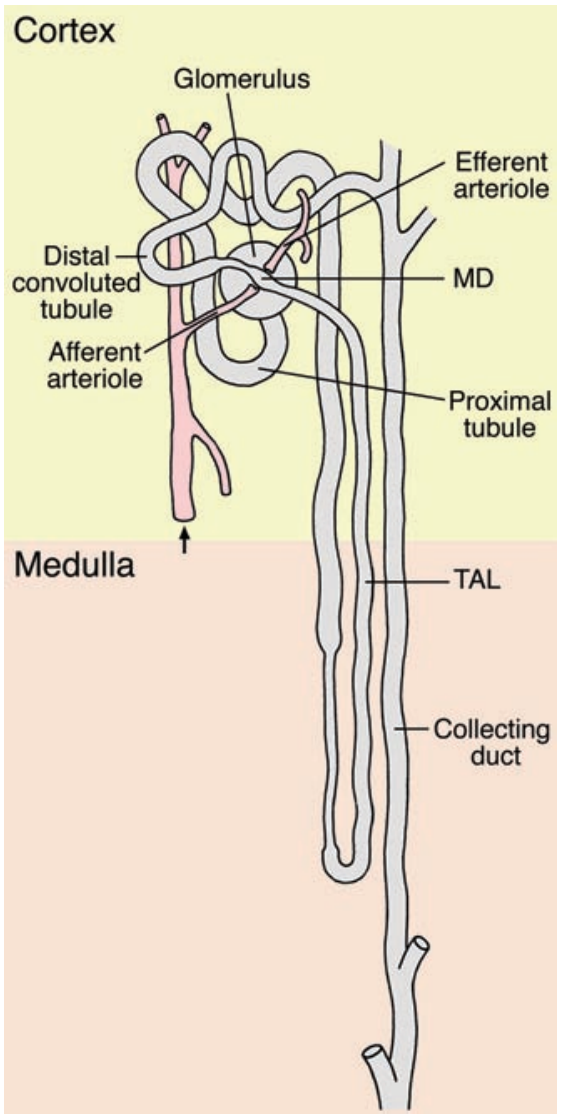

feedback mechanism (15). Like adenosine, ATP is able to constrict afferent arterioles without having a clear effect in postglomerular vessels (16). Consistent with the notion that there is a role for ATP in the autoregulatory response is the observation that mice lacking $\mathrm{P} 2 \mathrm{X} 1$ receptors, one type of ATP-selective $\mathrm{P} 2$ receptors, have an impaired capacity to autoregulate renal vascular resistance (17). The important finding that an increase in luminal $\mathrm{NaCl}$ concentration causes an increase in the release of ATP across the basolateral membrane of MD cells has been taken as further support that ATP acts as the mediator of the feedback response (18).

\section{Figure 2}

Schematic showing the structural and functional relationships within the JGA. The MD cells are shown in brown, the extraglomerular mesangium in blue, the VSMCs in magenta, the renin-producing cells in green, the fibroblasts of the adjacent interstitium in yellow, and the blood vessels in red. Note that both signaling pathways from the MD pass the extraglomerular mesangium, either to reach the VSMCs (I) to regulate filtration or the reninproducing cells (II) to mediate renin secretion.

\section{Figure 1}

Structural organization of the nephron. Blood vessels are shown in red; the glomerulus and the tubular system in gray. The afferent arteriole feeds the glomerular capillary network, where filtration of the blood occurs. The glomerular filtrate is processed as it travels through the different tubular segments. At the cell plaque of the MD, with which the TAL touches the vascular pole of its glomerulus, tubular fluid composition is sensed and paracrine signals are conveyed to smooth muscle and renin-producing cells of the afferent arteriole.

\section{Interaction between ATP and adenosine}

The observations of Castrop et al. reported in this issue of the JCI (6) make an important step towards resolving the perceived discrepancy. These authors have generated ecto-5'-nucleotidase-deficient mice and have used these mice to determine the role of extracellular AMP hydrolysis in tubuloglomerular feedback using micropuncture techniques. Ecto-5'-nucleotidase is a membrane-anchored enzyme that is predominantly responsible for the generation of adenosine from the nucleotide precursor $5^{\prime}$-AMP. This enzyme is highly expressed in the kidney and has been shown to be associated with various cells at the glomerular vascular pole (extraglomerular mesangial cells and fibroblasts, depending on species) (19). Its location is compatible with the suggested role of this enzyme in the local generation of adenosine in the extraglomerular mesangium and adjacent interstitium. Castrop et al. report that ecto-5' -nucleotidaseknockout mice have a markedly attenuated vasoconstrictor response to an increase in luminal $\mathrm{NaCl}$ concentration. Previous stud- ies using pharmacological inhibition of 5'nucleotidase have already suggested a role for this enzyme in tubuloglomerular feedback (20). Thus, if adenosine is required for $\mathrm{NaCl}$-dependent vasoconstriction, as the studies in A1AR-knockout mice suggest, hydrolysis of 5'-AMP catalyzed by ecto-5'nucleotidase appears to be the dominant mechanism of adenosine generation.

The observations by Castrop et al. (6) could be extended to include ATP, thereby providing a potential link between the ATP and adenosine hypotheses of tubuloglomerular feedback regulation. One may assume a chain of events that begins with the release of ATP by MD cells and that leads through a series of dephosphorylation steps to the generation of adenosine. In such a scenario, the rate of adenosine formation may vary as a function of the rate of ATP release. ATP dephosphorylation to $5^{\prime}$-AMP is catalyzed primarily by a family of enzymes called NTPDases (ecto-nucleoside triphosphate diphosphohydrolases), but several other enzymes may be involved (21).

Overall, the study of Castrop et al. (6) adds to the evidence that purinergic signal- 
ing is critical for the information transfer across the juxtaglomerular interstitial space. The results confirm the notion that adenosine generation is necessary for the vascular response. Furthermore, they strongly suggest that adenosine is formed in the extracellular space by nucleotide hydrolysis. While the data from Castrop et al. only directly invoke $5^{\prime}$-AMP as an adenosine precursor, it is not farfetched to assume that adenosine is the hydrolysis product of ATP and that its formation rate may well be modulated by varying levels of ATP. In this case, rather than being mediated by ATP or adenosine along alternative pathways, tubuloglomerular feedback may be the result of successive involvement of both ATP and adenosine. This may serve as a reminder that in long-standing scientific controversies, it is perhaps more the rule than the exception that both sides reflect part of the truth, and that the solution lies in combining these parts of the truth into a coherent picture.

Address correspondence to: Wilhelm Kriz, Department of Anatomy and Cell Biology, University of Heidelberg, Im Neuenheimer Feld 307, 69120 Heidelberg, Germany. Phone: 49-6221-54-8680; Fax: 49-6221-54-4951; E-mail: wilhelm.kriz@urz.uni-heidelberg.de.
1. Goormaghtigh, N. 1937. L'appareil neuro-myoartériel juxta-glomérulaire du rein: ses réactions en pathologie et ses rapports avec le tube urinifere. C. $R$. Seances Soc. Biol. Fil. 124:293-296.

2. Schnermann, J., and Briggs, J. 2000. Function of the juxtaglomerular apparatus: Control of glomerular hemodynamics and renin secretion. In The kidney: physiology and pathophysiology. D.W. Seldin and G. Giebisch, editors. Lippincott Williams \& Wilkins. Philadelphia, Pennsylvania, USA. 945-980.

3. Peti-Peterdi, J., et al. 2003. Luminal $\mathrm{NaCl}$ delivery regulates basolateral $\mathrm{PGE}_{2}$ release from macula densa cells. J. Clin. Invest. 112:76-82. doi:10.1172/ JCI200318018.

4. Paliege, A., et al. 2004. Inhibition of nNOS expression in the macula densa by COX-2-derived prostaglandin $\mathrm{E}_{2}$. Am. J. Physiol. Renal Physiol. 287:F152-F159.

5. Schweda, F., Kammerl, M., Wagner, C., Krämer, B., and Kurtz, A. 2004. Upregulation of macula densa cyclooxygenase-2 expression is not dependent on glomerular filtration. Am. J. Physiol. Renal Physiol. 287:F95-F101.

6. Castrop, H., et al. 2004. Impairment of tubuloglomerular feedback regulation of GFR in ecto-5'nucleotidase/CD73-deficient mice. J. Clin. Invest. 114:634-642. doi:10.1172/JCI200421851.

7. Schnermann, J. 2002. Adenosine mediates tubuloglomerular feedback. Am. J. Physiol. Regul. Integr. Comp. Physiol. 283:R276-R277; discussion R278-R279.

8. Nishiyama, A., and Navar, L. 2002. ATP mediates tubuloglomerular feedback. Am. J. Physiol. Regul. Integr. Comp. Physiol. 283:R273-R275; discussion R278-R279.

9. Osswald, H., Hermes, H., and Nabakowski, G. 1982. Role of adenosine in signal transmission of tubuloglomerular feedback. Kidney Int. Suppl. 12:S136-S142.

10. Osswald, H., Nabakowski, G., and Hermes, H. 1980. Adenosine as a possible mediator of metabolic control of glomerular filtration rate. Int. J. Biochem. 12:263-267.

11. Schnermann, J., Weihprecht, H., and Briggs, J. 1990.
Inhibition of tubuloglomerular feedback during adenosine 1 receptor blockade. Am. J. Physiol. Renal Physiol. 258:F553-F561.

12. Hansen, P., Castrop, H., Briggs, J., and Schnermann, J. 2003. Adenosine induces vasoconstriction through Gi-dependent activation of phospholipase $\mathrm{C}$ in isolated perfused afferent arterioles of mice. J. Am. Soc. Nephrol. 14:2457-2465.

13. Brown, R., et al. 2001. Abolished tubuloglomerular feedback and increased plasma renin in adenosine $A$ 1 receptor-deficient mice. Am. J. Physiol. Regul. Integr. Comp. Physiol. 281:R1362-R1367.

14. Sun, D., et al. 2001. Mediation of tubuloglomerular feedback by adenosine: Evidence from mice lacking adenosine 1 receptors. Proc. Natl. Acad. Sci. U. S. A. 98:9983-9988.

15. Inscho, E., Cook, A., and Navar, L. 1996. Pressuremediated vasoconstriction of juxtamedullary afferent arterioles involves P2-purinoceptor activation. Am. J. Physiol. 271:F1077-F1085.

16. Inscho, E. 2001. P2 receptors in regulation of renal microvascular function. Am. J. Physiol. Renal Physiol. 280:F927-F944.

17. Inscho, E.W., Cook, A.K., Imig, J.D., Vial, C., and Evans, R.J. 2003. Physiological role for $\mathrm{P}_{2} \mathrm{X}_{1}$ receptors in renal microvascular autoregulatory behavior. J. Clin. Invest. 112:1895-1905. doi:10.1172/JCI200318499.

18. Bell, P., et al. 2003. Macula densa cell signaling involves ATP release through a maxi anion channel. Proc. Natl. Acad. Sci. U. S. A. 100:4322-4327.

19. Le Hir, M., and Kaissling, B. 1993. Distribution and regulation of renal ecto-5'-nucleotidase: implications for physiological functions of adenosine. Am. J. Physiol. 264:F377-F387.

20. Thomson, S., Bao, D., Deng, A., and Vallon, V. 2000. Adenosine formed by 5 '-nucleotidase mediates tubuloglomerular feedback. J. Clin. Invest. 106:289-298.

21. Zimmermann, H. 2000. Extracellular metabolism of ATP and other nucleotides. Naunyn Schmiedebergs Arch. Pharmacol. 362:299-309.

\title{
Why does diabetes increase atherosclerosis? I don't know!
}

\author{
Ira J. Goldberg \\ Department of Medicine, Columbia University College of Physicians and Surgeons, New York, New York, USA.
}

\begin{abstract}
There is a wealth of clinical data showing the relationship between diabetes mellitus and atherosclerosis and its clinical complications. To dissect this relationship, investigators have attempted, usually unsuccessfully, to create a small-animal model in which diabetes accelerates vascular lesion development. This effort has often been complicated by development of hyperlipidemia leading to difficulty in differentiating the effects of hyperglycemia from those of lipid abnormalities. A study in the current issue of the JCI provides data on a new mouse model in which atherosclerosis initiation is accelerated in diabetic mice and is reduced by insulin therapy. Moreover, these animals have greater intra-arterial hemorrhage, which might be due to less stable plaques (see the related article beginning on page 659).
\end{abstract}

Nonstandard abbreviations used: AGE, advanced glycation end-product.

Conflict of interest: The author has declared that no conflict of interest exists.

Citation for this article: J. Clin. Invest. 114:613-615 (2004). doi:10.1172/JCI200422826.
Epidemiological data has firmly established the correlation between coronary heart disease and both type 1 and type 2 diabetes mellitus. Is the acceleration of atherosclerosis with insulin deficiency and insulin resistance due to defective insulin actions, hyperglycemia, or some other process that is present in diabetic patients? Several major glucose-lowering trials in type 1 (1) and type 2 diabetic (2) patients that showed reductions in microvascular complications failed to achieve significant reduction in macrovascular events. One hypothesis to explain these trial results is that metabolic abnormalities associated with diabetes, and not overt hyperglycemia per se, accelerate macrovascular complications. Support for this comes from the observation that coronary heart disease is increased in prediabetic patients (3): people who have several metabolic abnormalities associated with type 2 diabetes but who do not have fasting hyperglycemia or elevated glycosylated hemoglobin. Another possibility is that 